\title{
DIVERSIDADE DE ÁCAROS (ARACHNIDA: ACARI) EM CINCO CULTIVARES DE DUAS ESPÉCIES DE CAFEEIROS (COFFEA SPP.) EM GARÇA, ESTADO DE SÃO PAULO
}

\author{
J.L. de C. Mineiro ${ }^{1 *}$, M.E. Sato ${ }^{1}$, A. Raga ${ }^{1}$, V. Arthur ${ }^{2}$, K.G. Cangani ${ }^{1}$, F.V. Barbosa ${ }^{1}$
}

${ }^{1}$ Instituto Biológico, Centro Experimental Central, CP 70, CEP 13001-970, Campinas, SP, Brasil. E-mail: jefmin@hotmail.com

RESUMO

O presente estudo teve como objetivo caracterizar a diversidade de ácaros em cinco diferentes cultivares de duas espécies de cafeeiros, no Município de Garça, SP. Foram estudadas as seguintes espécies e cultivares de cafeeiro: Coffea canephora Pierre ex Froehner cv. Apoatã e Coffea arabica L. cultivares Mundo Novo, Icatu Vermelho, Icatu Amarelo e Catuaí Amarelo. Os ácaros foram obtidos de folhas, amostradas do terço médio em 10 plantas ao acaso de cada cultivar. A amostragem foi realizada mensalmente, entre abril de 2001 e junho de 2003. Apoatã foi a cultivar que apresentou a maior riqueza de espécies e o maior número de espécimes na superfície das folhas. Por outro lado, essa mesma cultivar foi a que apresentou o menor número de espécies e de espécimes no interior das domácias. A cultivar Icatu Vermelho foi a que apresentou a maior uniformidade na distribuição das espécies de ácaros enquanto a cultivar Apoatã a que apresentou a menor. Na superfície das folhas, as espécies fitófagas Brevipalpus phoenicis (Geijskes) eOligonychus ilicis (McGregor) foram muito abundantes e freqüentes em todas as cultivares, enquanto os predadores Euseius citrifolius Denmark \& Muma e E. concordis (Chant), ambos Phytoseiidae, foram os mais abundantes e freqüentes. Nas domácias, os ácaros mais freqüentes foram os predadores da família Stigmaeidae, sendo Zetzellia malvinae Matioli, Ueckermann \& Oliveira mais abundante em todas as cultivares de C. arabica e Agistemus brasiliensis Matioli, Ueckermann \& Oliveira muito abundante apenas na cultivar Icatu Vermelho.

PALAVRAS-CHAVE: Controlebiológico,manejodepragas,Phytoseiidae,Tetranychidae,Stigmaeidae.

\section{ABSTRACT}

DIVERSITY OF MITES (ARACHNIDA: ACARI) ON FIVE CULTIVARS OF TWO SPECIES OF COFFEE (COFFEA SPP.) IN GARÇA, STATE OF SÃO PAULO, BRAZIL. The aim of this study was to characterize the diversity of mites present on five different cultivars of two species of coffee in Garça county, state of São Paulo, Brazil. The following species and cultivars of coffee plant were studied: Coffea canephora Pierre ex Froehner cv Apoatã and C. arabica L. of cultivars Mundo Novo, Icatu Vermelho, Icatu Amarelo and Catuaí Amarelo. The mites were obtained from leaves collected from the mid-third of plants randomly chosen from each cultivar. The samples were collected monthly from April 2001 to June 2003. A poatã was the cultivar which presented the highest species richness and the highest number of specimens on the leaf surface. On the other hand, this cultivar presented the lowest number of species and of specimens inside domatia. Icatu Vermelho was thecultivar which presented the highest uniformity of distribution of mite species, and Apoatã was the cultivar with the lowest uniformity. On the leaf surface, the phytophagous species Brevipalpus phoenicis (Geijskes) and Oligonychus ilicis (McGregor) were the most abundant and frequent species in all cultivars. Among the predators, Euseiuscitrifolius Denmark \& Muma and E.concordis (Chant), both Phytoseiidae, were the most abundant and frequent on the leaf surface. The most frequent mites inside domatia were the predators of the family Stigmaeidae, in which Zetzellia malvinae Matioli, Ueckermann \& Oliveira were the most abundant in all cultivars of C. arabica. Agistemus brasiliensis Matioli, Ueckermann \& Oliveira were very abundant only within domatia of the cultivar Icatu Vermelho.

KEY WORDS: biological control, pest management, Phytoseiidae, Tetranychidae, Stigmaeidae.

\footnotetext{
${ }^{2}$ Universidade de São Paulo, Centro de Energia Nuclear na Agricultura, Laboratório de Irradiação de Alimentos e Radioentomologia, Piracicaba, SP, Brasil.

*Parte da Tese de Doutorado do primeiro autor defendida juntoà Universidade deSão Paulo/ Centro de Energia Nuclear na Agricultura, Piracicaba, SP, Brasil.
} 
Há cerca de 100 espécies vegetais descritas no gênero Coffea, sendo que somente duas produzem frutos que têm importância econômica no mercado internacional, Coffea arabica L. e Coffea canephoraPierre ex Froehner. Os produtos derivados dessas duas espécies são conhecidos como café arábica e café canéfora, respectivamente. O café arábica representa cerca de $70 \%$ do mercado internacional, por apresentar qualidade de bebida superior à de canéfora (PEREIRA \& SAKiYAma, 1999; Matiello et al., 2002).

Historicamente, ocafeeirotem tidoum papel-chave na economia e desenvolvimento social do Brasil. Nas últimas décadas, têm sido introduzidas novas cultivares decafeeiros, selecionadas pelo Instituto Agronômico de Campinas. Essas cultivares são responsáveis por cerca de $90 \%$ da produção de caféarábica (THOMAZIELLO et al., 2000; GUERREIRO FilHo et al., 2003).

Estudos sobre os ácaros que ocorrem no cafeeiro, em sua maioria, tratam de espécies fitófagas. Várias espécies deácaros-praga podem alcançar altos níveis populacionais, ocasionando perdas significativas aos produtores. Entre essas espécies estão Oligonychus ilicis (McGregor) (Tetranychidae) e Brevipalpus phoenicis (Geijskes) (Tenuipalpidae). Ácaros predadores, fungívoros e outros que fazem parte da acarofauna desta cultura são ainda pouco estudados (Pallini Filho et al., 1992; Reis et al., 2000; SpONGoski et al., 2005) no Brasil.

O estudo da estrutura da comunidade de ácaros no agroecossistema cafeeiro é fundamental para se compreender melhor as interações entre as diversas espécies presentes nas diferentes cultivares de cafeeiro, servindo de subsídio para a definição de programas de manejo integrado de pragas na cultura. $\mathrm{O}$ presente estudo teve como objetivo caracterizar a diversidade de ácaros presentes em cinco diferentes cultivares de duas espécies de cafeeiros.

\section{MATERIAL E MÉTODOS}

Apesquisasobreadiversidadedeácarosfoiconduzida na Estação Experimental "Alcides Carvalho" da Cooperativados CafeicultoresdeGarça-Garcafé,Municípiode Garça, SP ( $22^{\circ} 15^{\prime} \mathrm{S} ; 49^{\circ} 39^{\prime} \mathrm{O}, 682 \mathrm{~m}$ de altitude). Foram estudadas as seguintes espécies e cultivares de cafeeiro: C. canephora cv Apoatã IAC 2258 e C. arabica cultivares MundoNovoIAC388-17, Icatu Vermelho IAC 4045, Icatu Amarelo IAC 2944 e Catuaí Amarelo IAC 62.

$\mathrm{Na}$ área estudada de $\mathrm{C}$. canephora cv. Apoatã havia 447 plantas que foram plantadas em novembro de 1990, sendoduas plantas por cova, comintervalos entre covas de2meentrelinhas de $3,5 \mathrm{~m}$. NaáreacomMundoNovo, havia 473 plantas, queforam plantadas em setembrode 1978, sendo 2 plantas por cova, com espaçamento entre covas de $2 \mathrm{~m}$ e entre linhas de $4,0 \mathrm{~m}$. A área da cultivar
Icatu Vermelhocontavacom844 plantas, cujo plantiofoi em maio de 1983. Na área da cultivar de Icatu Amarelo, havia 657 plantas, com plantio em março de 1990. Na área de Catuaí Amarelo, havia 402 plantas, que foram plantadas em setembro de 1989. Ointervalo entre covas era de 1,5 m e entre linhas de $4 \mathrm{~m}$ e havia apenas uma planta por cova das cultivares Icatu Vermelho, Icatu Amarelo e Catuaí Amarelo.

Segundo o sistema de classificação de Köeppen, o clima de Garça é do tipo Cwa, clima quente, com inverno seco com menos de $30 \mathrm{~mm}$ de chuva no mês mais seco. A temperatura média do mês mais quente é superior a $22^{\circ} \mathrm{C}$ e do mês mais frio, inferior a $18^{\circ} \mathrm{C}$ (SeTZer, 1966).

As amostragens foram realizadas mensalmente e tiveram início em abril de 2001 e término em junho de 2003. Foram coletadas folhas do terço médio de 10 plantas escolhidas ao acaso. As folhas foram coletadas do terceiro ou quarto par, a partir da extremidade distal do ramo, tomando-se 12 folhas por planta (PALLinI FiLHo etal., 1992; ReIs etal., 2000; SPONGOSKI et al., 2005). As amostras foram colocadas em sacos de papel e acondicionadas em caixas de poliestireno contendo gelo para diminuir a atividade dosácaros. Asextrações dos ácaros foram realizadas na própria Estação Experimental, em Garça. Para tanto, as folhas decada planta foram imersas durante 5 min em uma solução deálcool a70\%. Em seguida, cada folha foi agitada nesta solução para desalojar os ácaros, passando-se então a solução por uma peneira com malha de $0,038 \mathrm{~mm}$. Os ácaros retidosna peneira foramarmazenadosemálcoola $70 \%$ atéa montagem em lâminas de microscopia para identificação. Para a coleta dos ácaros das domácias, cada folha foi posteriormente cortada ao longo da nervura central, mantendo-se um espaço de aproximadamente $0,5 \mathrm{~cm}$ de cada lado. As secções das folhas contendo a nervura central foram acondicionadas em frascos contendo álcool a 70\% para posterior exame.

Nos laboratórios do Centro Experimental Central do Instituto Biológico, em Campinas, SP, foram feitas as triagens dos ácaros coletados das folhas e armazenados em frascos com álcool a $70 \%$. As domácias foram contadas e abertas uma a uma com o auxílio de um bisturi para a retirada dos ácaros presentes (Pemberton \& Turner, 1989; Spongoski et al., 2005). Todos os ácaros encontrados foram montados em lâminas de microscopia, em meio de Hoyer. Uma amostra representativa das espécies encontradas foi depositada na coleção de referência de ácaros do Laboratório de Entomologia Econômica do Instituto Biológico (LEE/IB).

Para a análise da diversidade e uniformidade da acarofauna cafeeira, foram aplicados os índices de Shannon-Wiener e dePielou, respectivamente. Foram analisadas dominância (superdominante, dominante e não-dominante), abundância (superabundante, 
muito abundante, abundante, comum, dispersa e rara), freqüência (superfreqüente, muito freqüente, freqüente e pouco freqüente) e constância (constante, acessória e acidental). Os intervalos foram definidos através dos intervalos de confiança das estatísticas utilizadas $(p=0,05$ e $p=0,01)$. Na realização dessas análises foi utilizado o programa ANAFAU desenvolvido pelo Setor de Entomologia da ESALQ/USP (MorAes et al., 2003). Foi utilizado o índice de similaridadedeMorisita-Horn $\left(\mathrm{C}_{\mathrm{MH}}\right)$ (MAGURRAN, 1988) para estabelecer o grau de semelhança entre as diferentes cultivares e nas diferentes partes das plantas amostradas (superfície das folhas e domácias).

\section{RESULTADOS}

Foram coletados ao todo 14.040 ácaros nas cinco diferentes cultivares estudadas (Tabela 1). Na superfície das folhas, foram registradas 63 espécies de ácaros pertencendo a 28 famílias. Phytoseiidae foi a família que apresentou o maior número de espécies, 11 ao todo, seguida pelas famílias Tydeidae e Tarsonemidae, ambas com cinco, e Cheyletidae, com quatro (Tabela 2). No interior das domácias foram encontradas 17 espécies distribuídas em nove famílias. Tydeidae foi a família com o maior número de espécies, cinco ao todo, seguida pela família Phytoseiidae, com três (Tabela 3).

Superfície das folhas. A maior riqueza de espécies foi observada na cultivar Apoatã, seguida pelas cultivares Icatu Amarelo, Mundo Novo, Icatu Vermelho e Catuaí Amarelo (Tabela 1). O número de espécimes não seguiu a mesma tendência, com maior quantidade em Apoatã, seguida por Catuaí Amarelo, Icatu Vermelho, Icatu Amarelo e Mundo Novo.

Os maiores índices de diversidade foram obtidos para Icatu Vermelho e Mundo Novo, e o menor para Apoatã (Tabela 1). Com relação à uniformidade de espécies, o maior índice foi observado para Icatu
Vermelho, onde a relação entre a primeira espécie dominante, $O$. ilicis, e a segunda, B. phoenicis, ficou em torno de 1,2 vezes, com distribuição mais uniforme para as outras espécies dominantes. A cultivar Apoatã foi a que apresentou o mínimo de uniformidade e o máximo de dominância e a relação entre a primeira espécie dominante, B. phoenicis, e a segunda, Lorryia sp.1(Tydeidae), foi de3,9vezes, sendosuperior ao das demais cultivares. A grande maioria das espécies, $71 \%$, apresentou poucos representantes (Tabelas 1e2).

Domácias. Nas domácias, a maior riqueza de espécies foi constatada nas cultivares Mundo Novoe Icatu Vermelho (Tabela 1). Com relação ao número de espécimes, o maior foi observado na cultivar Mundo Novo (1.189) e o menor na cultivar Apoatã (151). A riqueza de espécies nas domácias foi sempre inferior à observada na superfície das folhas em todas as cultivares. $\mathrm{O}$ número de espécies no interior das domácias variou de sete (Catuaí Amarelo) a 11 (Mundo Novo Icatu Vermelho), enquanto que o número de espécies presentes na superfície das folhas oscilou entre 25 (Catuaí Amarelo) e 38 (Apoatã).

O maior índice de diversidade foi obtido para Icatu Vermelho (1,53), diferenciando marcadamente das demais cultivares (Tabela 1). Com relaçãoà uniformidade, foi observado o maior índice em Icatu Vermelho. A relação entre a primeira espécie dominante,Lorryia sp.1, e a segunda, Zetzellia malvinae Matioli, Ueckermann \& Oliveira (Stigmaeidae), foi em torno de 2,4 vezes. As cultivares Apoatã, Mundo Novo, Icatu AmareloeCatuaí Amarelo apresentaram baixa uniformidade e alta dominância, ou seja, a relação entre a primeira espécie dominante e a segunda foi elevada. Para Apoatã, essa relação foi de 19,6 vezes entre Lorryia sp.1 e B. phoenicis; para a Mundo Novo foi de 11,9 vezes, entre Lorryia sp.1 e Z. malvinae; para Icatu Amarelo foi de 12,4 vezes entre Lorryia sp.1 e Z. malvinae; e para Catuaí Amarelo foi de 7 vezes, entre Lorryia sp.1 e Z. malvinae (Tabela 3).

Espécies mais comuns na superfície das folhas. No geral, para todas as cultivares, B. phoenicis, O.ilicis,

Tabela 1 - Número de espécies e espécimes, e índices de diversidade (Shannon-Wiener) e uniformidade (Pielou) de espécies de ácaros nas diferentes cultivares de cafeeiro, no município de Garça, Estado de São Paulo. Período de abril de 2001 a junho de 2003.

\begin{tabular}{|c|c|c|c|c|c|c|c|c|c|c|}
\hline \multirow[t]{4}{*}{ Parâmetros avaliados } & \multicolumn{10}{|c|}{ Cultivares de cafeeiro } \\
\hline & \multirow{2}{*}{\multicolumn{2}{|c|}{$\frac{\text { Coffea canephora }}{\text { Apoatã }}$}} & \multicolumn{8}{|c|}{ Coffea arabica } \\
\hline & & & \multicolumn{2}{|c|}{ Mundo Novo } & \multicolumn{2}{|c|}{ Icatu Vermelho } & \multicolumn{2}{|c|}{ Icatu Amarelo } & \multicolumn{2}{|c|}{ Catuaí Amarelc } \\
\hline & folha & domácia & folha & domácia & folha & domácia & folha & domácia & folha & domácia \\
\hline Número de espécies & 38 & 9 & 29 & 11 & 27 & 11 & 32 & 10 & 25 & 7 \\
\hline Número de espécimes & 4.099 & 151 & 1.120 & 1.189 & 1.712 & 258 & 1.644 & 966 & 1.987 & 914 \\
\hline Índice de diversidade & 1,39 & 0,46 & 2,01 & 0,52 & 2,08 & 1,53 & 1,92 & 0,43 & 1,74 & 0,45 \\
\hline Índice de uniformidade & 0,38 & 0,21 & 0,60 & 0,21 & 0,63 & 0,64 & 0,55 & 0,19 & 0,54 & 0,23 \\
\hline
\end{tabular}


Tabela 2 - Análise faunística para os ácaros encontrados em folhas de Coffea canephora e C. arabica, no município de Garça, Estado de São Paulo. Período de abril de 2001 a junho de 2003.

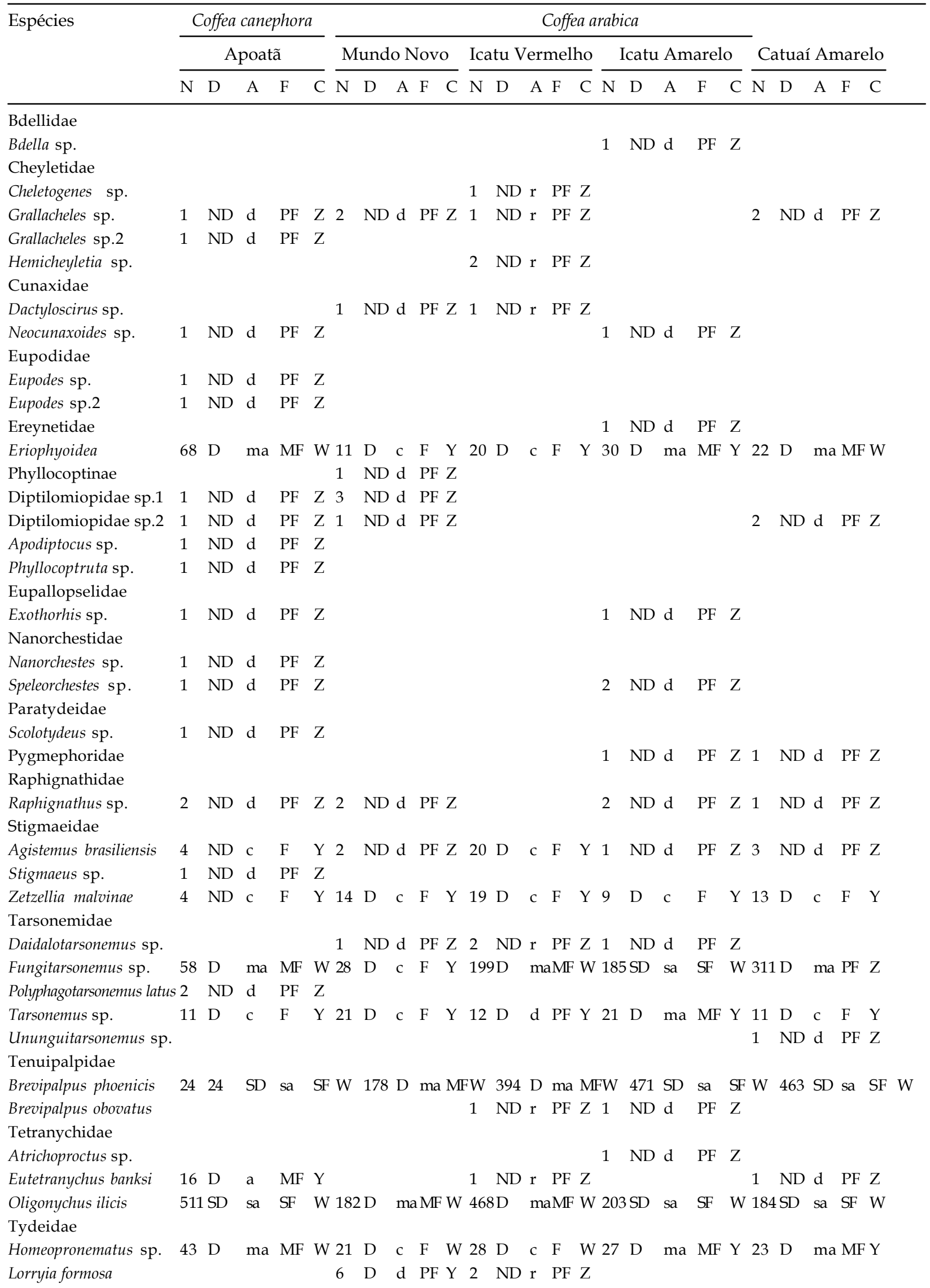


Tabela 2 - Continuação.

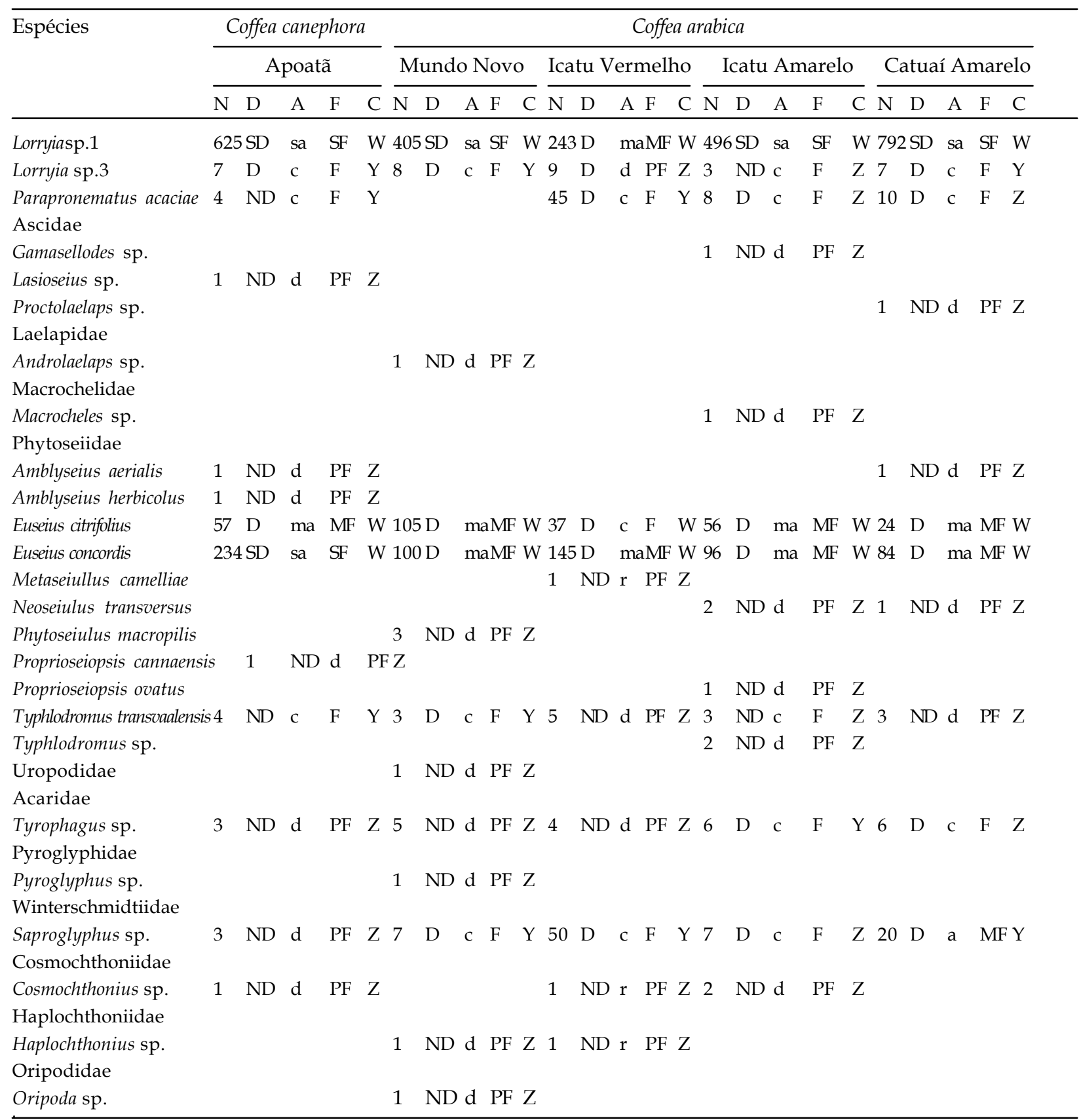

$\mathrm{N}=$ total de indivíduos

$\mathrm{D}=$ dominância: SD: superdominante; D: dominante; ND: não dominante.

A = abundância: sa: superabundante; ma: muito abundante; a: abundante; c: comum; d: dispersa; r: rara.

$\mathrm{F}=$ freqüência: $\mathrm{SF}$ : superfreqüente; $\mathrm{MF}$ : muito freqüente; $\mathrm{F}$ : freqüente; $\mathrm{PF}$ : pouco freqüente.

$\mathrm{C}=$ constância: $\mathrm{W}$ : constante; $\mathrm{Y}$ : acessória; $\mathrm{Z}$ : acidental.

Lorryia sp.1 e Euseius concordis (Chant) (Phytoseiidae) foram as espécies mais abundantes e freqüentes. Entre os fitoseídeos, Euseius citrifolius Denmark \& Muma e E. concordis foram dominantes em todas as cultivares. Entre os estigmeídeos, Agistemus brasiliensis Matioli, Ueckermann \& Oliveira foi freqüente e dominante apenas em Icatu Vermelho, enquanto que $Z$. malvinae foi dominante em todas as cultivares de arábica. Na cultivar Apoatã foram encontrados poucos espécimes destas duas espécies de estigmeídeos (Tabela 2).

Espécies mais comuns nas domácias. Lorryiasp.1 foi a espécie mais abundante e freqüente em todas as cultivares avaliadas. O tenuipalpídeo B. phoenicis foi encontrado na maioria dascultivares, porém em quantidade muito baixa. Na cultivar Catuaí Amarelo não 
Tabela 3 - Análise faunística para os ácaros encontrados em domácias de folhas de Coffea canephora e C. arabica, no município de Garça, Estado de São Paulo. Período de abril de 2001 a junho de 2003.

\begin{tabular}{|c|c|c|c|c|c|c|c|c|c|c|c|c|c|c|c|c|c|c|c|c|}
\hline \multirow[t]{3}{*}{ Espécies } & \multirow{2}{*}{\multicolumn{4}{|c|}{$\frac{\text { Coffea canephora }}{\text { Apoatã }}$}} & \multicolumn{16}{|c|}{ Coffea arabica } \\
\hline & & & & & \multicolumn{4}{|c|}{ Mundo Novo } & \multicolumn{4}{|c|}{ Icatu Vermelho } & \multicolumn{5}{|c|}{ Icatu Amarelo } & \multicolumn{3}{|c|}{ Catuaí Amarelo } \\
\hline & $\mathrm{N}$ & $\mathrm{D}$ & A F & $\mathrm{C}$ & $\mathrm{N}$ & $\mathrm{D} A$ & $\mathrm{~F}$ & $\mathrm{C}$ & $\mathrm{N}$ & $\mathrm{D}$ & A & $\mathrm{F}$ & $\mathrm{C} N$ & $\mathrm{~N} \quad \mathrm{D}$ & $\mathrm{A}$ & $\mathrm{F}$ & $\mathrm{CN}$ & $\mathrm{N}$ & D A F & $\mathrm{C}$ \\
\hline \multicolumn{21}{|l|}{ Bdellidae } \\
\hline $\begin{array}{l}\text { Bdella sp. } \\
\text { Stigmaeidae }\end{array}$ & & & & & 2 & NDd & PF & Y & & & & & & & & & & 2 & ND c F & $\mathrm{Y}$ \\
\hline Agistemus brasiliensis & 1 & ND & c F & $\mathrm{Y}$ & 7 & $\mathrm{D} C$ & $\mathrm{~F}$ & $\mathrm{Y}$ & $42 \mathrm{I}$ & $\mathrm{D} \quad \mathrm{r}$ & ma & MF Y & Y & & & & & 3 & $\mathrm{ND}$ a $\mathrm{MF}$ & \\
\hline $\begin{array}{l}\text { Zetzellia malvinae } \\
\text { Tarsonemidae }\end{array}$ & 1 & ND & c F & $\mathrm{Y}$ & 88 & SD sa & SF & W & $47 \mathrm{I}$ & D 1 & ma $\mathrm{I}$ & MF $\mathrm{W}$ & W 70 & 0 SD & D sa & SF & W 1 & $113 \mathrm{~S}$ & SD sa SF & W \\
\hline Daidalotarsonemus sp. & & & & & & & & & 1 & ND & d & PF Z & Z & & & & & & & \\
\hline $\begin{array}{l}\text { Tarsonemus sp. } \\
\text { Tenuipalpidae }\end{array}$ & & & & & 1 & $\mathrm{NDd}$ & $\mathrm{PF}$ & $\mathrm{Z}$ & 2 & ND & d & PF $\quad \mathrm{Z}$ & Z 1 & ND & $\mathrm{D} c$ & $\mathrm{~F}$ & Y & & & \\
\hline $\begin{array}{l}\text { Brevipalpus phoenicis } \\
\text { Tetranychidae }\end{array}$ & 7 & $\mathrm{D}$ & a $\mathrm{MF}$ & Y & 7 & $\mathrm{D} c$ & $\mathrm{~F}$ & $\mathrm{Y}$ & 6 & D c & c & $\mathrm{F}$ & Y 11 & $1 \mathrm{D}$ & $\mathrm{ma}$ & MF & $Y$ & & & \\
\hline $\begin{array}{l}\text { Oligonychus ilicis } \\
\text { Tydeidae }\end{array}$ & & & & & & & & & & & & & 1 & $\mathrm{ND}$ & D c & $\mathrm{F}$ & Y & & & \\
\hline Homeopronematus sp. & & & & & 19 & D ma & $\mathrm{MF}$ & $\mathrm{Y}$ & $39 \mathrm{I}$ & D 1 & ma $\mathrm{I}$ & MF Y & Y 7 & $\mathrm{D}$ & $\mathrm{ma}$ & MF & $Y 3$ & 3 & $\mathrm{ND} \mathrm{c} \quad \mathrm{F}$ & $\mathrm{Y}$ \\
\hline Lorryia formosa & 1 & ND & c F & $\mathrm{Y}$ & 6 & $\mathrm{D} c$ & $\mathrm{~F}$ & $\mathrm{Z}$ & 1 & ND & d & PF Z & Z 3 & ND & D c & $\mathrm{F}$ & Y 1 & 1 & $\mathrm{ND} d \mathrm{PF}$ & $\mathrm{Y}$ \\
\hline $\begin{array}{l}\text { Lorryia sp.1 } \\
\text { Lorryia sp.2 }\end{array}$ & 137 & SD & sa SF & $\mathrm{W}$ & $\begin{array}{l}1050 \\
1\end{array}$ & $\begin{array}{l}\text { SD sa } \\
\text { NDd }\end{array}$ & $\begin{array}{l}\text { SF } \\
\mathrm{PF}\end{array}$ & $\begin{array}{l}\mathrm{W} \\
\mathrm{Z}\end{array}$ & 114 & $\mathrm{SD} s$ & sa $s$ & SF $\mathrm{h}$ & W 86 & $69 \mathrm{SD}$ & D sa & SF & W 7 & $791 \mathrm{~S}$ & SD sa SF & W \\
\hline $\begin{array}{l}\text { Parapronematus acacio } \\
\text { Phytoseiidae }\end{array}$ & & & & & & & & & & 2 & ND & d & PF Z & & & & & 1 & $\mathrm{ND}$ d PF & Y \\
\hline Euseius citrifolius & & & & & 5 & $\mathrm{NDc}$ & $\mathrm{F}$ & $\mathrm{Y}$ & & & & & 1 & ND & $\mathrm{D} c$ & $\mathrm{~F}$ & Y & & & \\
\hline Euseius concordis & 1 & ND & c F & $\mathrm{Y}$ & 3 & $\mathrm{NDc}$ & $\mathrm{F}$ & $\mathrm{Y}$ & & & & & 1 & ND & $\mathrm{D} c$ & $\mathrm{~F}$ & Y & & & \\
\hline $\begin{array}{l}\text { Typhlodromus camellia } \\
\text { Acaridae }\end{array}$ & & & & & & & & & & 1 & ND c & d & PF Z & & & & & & & \\
\hline $\begin{array}{l}\text { Tyrophagus sp. } \\
\text { Winterschmidtiidae }\end{array}$ & 1 & ND & c F & $\mathrm{Y}$ & & & & & & & & & & & & & & & & \\
\hline Saproglyphus sp. & 1 & ND & c F & $\mathrm{Y}$ & & & & & 3 & ND & c & $\mathrm{F}$ & Z 2 & ND & D c & $\mathrm{F}$ & Y & & & \\
\hline
\end{tabular}

foi encontrado nenhum exemplar de B. phoenicis no interior das domácias. Dentre os ácaros predadores, poucos espécimes de fitoseídeos foram observados no interior dessasestruturas. Omaiornúmerodefitoseídeos (oito) foi registrado em Mundo Novo. As espécies de estigmeídeosforamasmaisfreqüentes, sendoZ.malvinae a espécie mais abundante em todas as cultivares de arábica. A. brasiliensis foi muito abundante apenas em Icatu Vermelho. Em Icatu Amarelo não foi encontrado nenhum espécime dessa espécie, enquanto na cultivar Apoatã foi registrado apenas um espécime de $A$. brasiliensis e um de Z. malvinae (Tabela 3).

Similaridade da composição de espécies nas diferentes cultivares

São apresentados os índices de similaridade de Morisita-Horn para as diferentes cultivares e partes da folha amostrada (limbo e domácias).
Superfície das folhas. Verificou-se que as composições de espécies de ácaros nas diferentes cultivares foram mais próximas entre si para: Mundo Novo e Icatu Amarelo; Mundo Novo e Catuaí Amarelo; e Icatu Amarelo e Catuaí Amarelo, com índices de similaridade entre 0,91 e 0,96. O menor índice $(0,59)$ foi observado entre Apoatã e Mundo Novo (Tabela 4).

Domácia. Verificou-se que as espécies encontradas em Apoatã, Mundo Novo, Icatu Amarelo e Catuaí Amarelo foram similares entre si. Contudo, as espécies encontradas em Icatu Vermelho apresentaram maiores contrastes em relação às encontradas em outras cultivares, com índices de similaridade iguais ou inferiores a 0,78 (Tabela 5).

\section{DISCUSSÃO}


Tabela 4 - Índice de similaridade (Morisita-Horn) para a composição de espécies de ácaros em folhas de Coffea canephora e C. arabica, no município de Garça, Estado de São Paulo. Período de abril de 2001 a junho de 2003.

\begin{tabular}{|c|c|c|c|c|c|}
\hline \multirow[t]{3}{*}{ Cultivares } & \multicolumn{5}{|c|}{ Cultivares de cafeeiro } \\
\hline & \multicolumn{2}{|c|}{ Coffea canephora } & \multicolumn{3}{|c|}{ Coffea arabica } \\
\hline & Apoatã & Mundo Novo & Icatu Vermelho & Icatu Amarelo & Catuaí Amarelo \\
\hline Apoatã & - & 0,59 & 0,71 & 0,79 & 0,67 \\
\hline Mundo Novo & - & - & 0,70 & 0,92 & 0,91 \\
\hline Icatu Vermelho & - & - & - & 0,80 & 0,67 \\
\hline Icatu Amarelo & - & - & - & - & 0,96 \\
\hline Catuaí Amarelo & - & - & - & - & - \\
\hline
\end{tabular}

Tabela 5 - Índice de similaridade (Morisita-Horn) para a composição de espécies deácaros em domácias deCoffea canephora e C. arabica, no município de Garça, Estado de São Paulo. Período de abril de 2001 a junho de 2003.

\begin{tabular}{lcccccc}
\hline Cultivares & \multicolumn{5}{c}{ Cultivares decafeeiro } \\
\cline { 2 - 4 } & \multicolumn{2}{c}{ Coffea canephora } & & \multicolumn{3}{c}{ Coffea arabica } \\
\cline { 2 - 4 } \cline { 5 - 7 } & Apoatã & Mundo Novo & & Icatu Vermelho & Icatu Amarelo & Catuaí Amarelo \\
\hline Apoatã & - & 1,0 & & 0,73 & 1,0 & 1,0 \\
Mundo Novo & - & - & 0,77 & 1,0 & 1,0 \\
Icatu Vermelho & - & - & - & 0,75 & 0,78 \\
Icatu Amarelo & - & - & - & - & 1,0 \\
Catuaí Amarelo & - & - & - & - & - \\
\hline
\end{tabular}

O estudo da acarofauna em diversas cultivares de cafeeiro indicou uma considerável diferença entre Apoatã e as cultivares de café arábica. Em Apoatã foi constatado o maior número de ácaros em folhas, com total superior ao dobro do observado em cada uma das outras cultivares. Porém, no caso das domácias, a situação foi oposta, observando-se o menor número de ácaros no interior dessas estruturas em Apoatã. $O$ maior contraste foi observado para Mundo Novo, que apresentou aproximadamente 7,9 vezes mais ácaros nas domácias do que Apoatã.

Apesar doelevado número de ácaros na superfície das folhas em Apoatã, o índice de diversidade foi relativamente baixo, sendo inferior ao das demais cultivares.

No caso de café arábica, a cultivar Icatu Vermelho mostrou que a distribuição das espécies foi mais uniforme em relação às outras cultivares, principalmente nas domácias. Isto refletiu também na diversidadeque, por conseqüência, foi também mais elevada que nas demais cultivares. Essas diferenças encontradas poderiam ser atribuídas às características genéticas das plantas. Entretanto, as cultivares Icatu Vermelho e Icatu Amarelo, que apresentaram diferenças consideráveis no número de espécimes e diversidade de ácaros presentes principalmente nas domácias, são ambas provenientes de cruzamentos entre $C$. canephora e C. arabica cv. Mundo Novo (TomazIElloet al., 2000; MAtielloetal., 2002). Embora geneticamente semelhantes, pequenas diferenças entre as domácias podem existir entre essas cultivares. Outros fatores, ainda não definidos, também podem ter contribuído para essas diferenças. Quanto aos fatores climáticos, as cultivares estavam sujeitas a condições semelhantes, uma vez que todo o conjunto de plantas estava em um mesmo local. $\mathrm{O}$ vigor e as condições nutricionais das plantas eram aparentemente semelhantes, pois recebiam os mesmos tratos culturais.

Nas folhas, a espécie claramente dominante entre os fitófagos, em todas as cultivares, foi B. phoenicis, especialmente na cultivar Apoatã. O grande número deácaros dessa espécie nesta cultivar pode ser devido à conformação diferente desta cultivar em relação às demais. As plantas de Apoatã apresentam porte mais alto, atingindo $4 \mathrm{a} 5 \mathrm{~m}$, com elevado número de hastes por planta (multicaule) (MATiEllo et al., 2002). As folhas também são maiores, com até $30 \mathrm{~cm}$ de comprimento. A menor diversidade de ácaros, principalmente de predadores, poderia ser outro fator responsável pela alta população de B. phoenicis em Apoatã. O número de ácaros estigmeídeos em Apoatã (incluindo superfície das folhas e domácias) foi bem inferior ao das outras cultivares, podendo ser um dos fatores importantes que podem ter influenciado nas populações de B. phoenicis em cafeeiro. A população total de fitoseídeos em Apoatã foi semelhanteou maior doque as das outras cultivares estudadas.

O tetraniquídeo $O$. ilicis, que ocupou a segunda posição, coexistindo com $B$. phoenicis em todas as 
cultivares estudadas, poderia ser considerado como espécie co-dominante. Nos estudos conduzidos em Minas Gerais por Pallini Filhoet al. (1992) eSPONGOSKI et al. (2005), esse mesmo padrão foi observado.

A ocorrência de fitoseídeos em cafeeiros é muito comum no Brasil. Dentre essas espécies, Iphiseiodes zuluagai Denmark \& Muma é muito comum nessa cultura (Flechtmann, 1967; Moraes et al., 1986 e 2004; PAllini Filho et al., 1992). Entretanto, essa espécie não foi constatada neste estudo realizado em Garça. As espécies E. citrifolius e E. concordis, que foram dominantes em todas as cultivares de cafeeiro em Garça, também foram encontradas em cafeeiros de Minas Gerais, porém, geralmente em menor densidade populacional (PALLINIFILHOetal., 1992; SPONGOSKI etal., 2005).

A presença de ácaros em domácias é bastante conhecida. Diversos autores mencionaram que as domácias podem servir de abrigo e refúgio para diversas espécies de ácaros (DedeccA, 1957; Pemberton \& TURNER, 1989; W ALTER\& O'DOWD, 1992; O'DOWD, 1994; WAlter, 1996; McMurtry \& CROFT, 1997; Gerson et al., 2003; Matos et al.,2004). Entretanto, poucoseconhece a respeito da função das domácias em cafeeiro. Este estudo realizado em diferentes cultivares de cafeeiros evidenciou alguns pontos. O primeiro foi a constatação de que algumas espécies de ácaros (comoLorryia sp.1 e Z. malvinae) estavam freqüentemente associadas a estas estruturas. $\mathrm{O}$ segundo ponto foi que a maioria das espécies encontradas nas domácias era principalmente de predadores, fungívoros e até uma combinação de ambos.

Nas domácias, a diversidade de ácaros variou muito entre as diferentes cultivares de arábica e de canéfora. No caso da Apoatã, a baixa diversidade de ácaros nas domácias e um número de espécimes sensivelmente menor do que nas outras cultivares se devem provavelmente ao formato destas estruturas. As domácias de Apoatã apresentaram-se de modo geral, como uma grandecavidaderasa, já as domácias de arábica possuíam uma câmara globular e uma abertura de formato arredondado a alongado. Devido a esse formato singular das domácias da cultivar Apoatã, essas não poderiam servir de abrigo ou refúgio para os ácaros encontrados nessas estruturas. Outros autores realizaram estudos sobre domácias de cafeeiros, principalmente em cultivares de arábica, observando que essas estruturas podem apresentar diferentes profundidades, com diversos formatos e tamanhos de abertura (BITANCOURT, 1927; DEDECCA, 1957; A dÂMOLIDE BARros, 1961; NAKAMURA et al., 1992; O'Dowd, 1994).

Das espécies de predadores encontradas no interior das domácias, $Z$. malvinae parece ser uma das mais bem adaptadas a viver nestas estruturas. Provavelmente, as domácias possam oferecer condições mais favoráveis à sobrevivência desta espécie em cafeeiro. A ocorrência de ácaros da família Stigmaeidae no interior de domácias é considerada bastante comum como relatado por outros autores (Pemberton \& Turner, 1989; O’Dowd, 1994; Walter, 1996).

Os fatores genéticos do hospedeiro e as condições ambientais podem atuar direta ou indiretamente sobre o desenvolvimento, reprodução e sobrevivência deácaros fitófagos, predadores ou mesmo micófagos. Além disso, aspectos nutricionais da planta, competições intra e interespecíficas entre ácaros e outros organismos, e muitos outros fatores podem influenciar no estabelecimento da complexa comunidade de ácaros que vivem sobre as plantas (JEPPSON et al., 1975; GERSON et al., 2003). Informações sobre as interações plantas-ácaros ainda são bastante escassas, não apenas para cafeeiro, mas também para a maioria das plantas cultivadas.

Estudos mais detalhados sobre a diversidade de ácaros em cafeeiros, em diferentes cultivares, em diferentes regiões são necessários para um melhor conhecimento das espécies presentes na cultura. Os resultados obtidos neste estudo indicam que a acarofauna em cafeeiro é muito diversificada e que a composição das espécies, principalmente de predadores, pode variar bastante de uma cultivar para outra. Estudos mais aprofundados sobre essas espécies, principalmente de predadores, poderiam gerar informações para o estabelecimento de um programa de manejo da cultura mais adequado, visando à manutenção do equilíbrio populacional de ácaros na cultura.

\section{AGRADECIMENTOS}

Ao Consórcio Brasileiro de Pesquisa e Desenvolvimento do Café e EMBRAPA Café pelo apoio financeiro à pesquisa; à CAPES pela concessão da bolsa de doutoradoao primeiroautor; ao CNPq pelas bolsas de produtividade em pesquisa; à Cooperativa dos Cafeicultores de Garça - Garcafé pelo apoio nos trabalhos de campo; ao Dr. Antonio C. Lofego pela confirmação das espécies de Phytoseiidae e Tarsonemidae; ao Dr. Maurício Zacarias pela confirmação das espécies de Tydeidae; à Dra. Denise Navia pela ajuda com os eriofídeos; ao Dr. Aníbal R. Oliveira pela ajuda com os oribatídeos; aos Profs. Dr. Carlos H. W. Flechtmann ESALQ/USP e Dr. Reinaldo Feres - UNESP/São José do Rio Preto pela confirmação dos gêneros e espécies de tetraniquídeosa; os estagiários Alex Carrijo, Fernando Sarreta, Márcio Mendonça, Guilherme Vianna e Giovani Almeida pelo auxílio nas coletas e montagem dos ácaros; ao Dr. Gilberto J. de Moraes pelas sugestões oferecidas no decorrer do trabalho e na redação do manuscrito. 
REFERENCIAS

ADÂMOLI DE BARros,M.A.Morfologia eanatomia das domácias em Coffea arabica L. Anais da Escola Superior de Agricultura "Luiz de Queiroz", v.17, p.165-206, 1961.

Bitancourt, A.A. A acarophilia do cafeeiro e seu papel eventual na defesa da planta contra os fungos parasitas. Boletim Biológico, v.10, p.203-208, 1927.

Dedecca, D.M. Anatomia e desenvolvimento ontogenético de Coffea arabica L. var. Typica Cramer. Bragantia, v.16, p.315-367, 1957.

Flechtmann, C.H.W. Os ácaros do cafeeiro. Anais da Escola Superior de Agricultura "Luiz de Queiroz", v.24, p.91-95, 1967.

Gerson, U.; Smiley, R.L.; OchoA, R. Mites (Acari) for pest control. Oxford: Blackwell Science, 2003.539p.

Guerreiro Filho, O.; Fazuoli, L.C.; Aguiar, A.T.E.Cultivares de Coffea arabica selecionadas peloIAC: características botânicas, tecnológicas, agronômicas e descritores mínimos. Agronômico, v. 55, p. 34-37, 2003.

Jeppson, L.R.; KeIFer, H.H.; BaKer, E.W. Mites injurious to economic plants. Berkeley: University of California Press, 1975. 614p.

MagurRan, A.E. Ecological diversity and its measurement. Princeton: Princeton University Press, 1988. 178p.

Matos, C.H.C.; Pallini, A.; Ghaves, F.F.; Galbiati, C. Domácias do cafeeiro beneficiam o ácaro predador Iphiseiodes zuluagai Denmark \& Muma (Acari: Phytoseiidae)? Neotropical Entomology, v.33, p.57-63, 2004.

Matiello, J.B.; Santinato, R.; Garcia, A.W.R.; Almeida, S.R.; FERNANDES, D.R. Cultura do caféno Brasil. Novo manual de recomendações. Rio de Janeiro: MAPA/PROCAFÉ FUNDAÇÃO PROCAFÉ, 2002. 387p.

McMurtry,J.A. \&C Roft, B.A. Life-styles of phytoseiid mites and their roles in biological control. Annual Review of Entomolology, v.42, p.291-321, 1997.

Moraes, R.C.B.;H addad, M.L.; Silveira Neto, S.; Reyes, A.E.L. Software para análise faunística - ANAFAU. In: SIMPÓSIO DE CONTROLE BIOLÓGICO, 8., 2003, São Pedro, SP. Resumos. São Pedro, 2003, p.195.

Moraes, G.J.de; McMurtry, J.A.; Denmark, H.A. A catalog of the mite family Phytoseiidae: references to taxonomy, synonymy, distribution and habitat. Brasília: EMBRAPA-DDT, 1986. 353p.

Moraes, G.J. DE; McMurtry, J.A.; Denmark, H.A.; Campos, C.B. A revised catalog of the mite family Phytoseiidae. Zootaxa, v.434, p.1-494, 2004.

NaKamura, T.; TANicughi, T.; MaEdA, E. Leaf anatomy of Coffea arabica $\mathrm{L}$. with reference to domatia. Japanese Journal of Crop Science, v.61, p.642-650, 1992.

O'DowD, D.J. Mite association with the leaf domatia of coffee (Coffea arabica) in north Queensland, Australia. Bulletin of Entomological Research, v.84, p.361-366, 1994.

Pallini Filho, A.; Moraes, G.J. De; Bueno, V.H.P. Ácaros associados ao cafeeiro (Coffeaarabica L.) no sul de Minas Gerais. Ciência e Prática, v.16, p.303-307, 1992.

Pemberton, R.W.;T urner, C.E. Occurrence of predatory and fungivorous mites in leaf domatia. American Journal of Botany, v.76, p.105-112, 1989.

Pereira, A.A. \& Sakiyama, N.S. Cultivares melhoradas de café arábica. In: ZAмBolim, L. (Ed.). I Encontro sobre produção de café com qualidade. Viçosa, 1999. p. 241-257.

ReIs,P.R.;Souza, J.C.DE;SouZA,E.O.;TEOdoro, A.V.Distribuição espacial do ácaro Brevipalpus phoenicis (Geijskes) (Acari: Tenuipalpidae) em cafeeiro (Coffea arabica L.). Anais da Sociedade Entomológica do Brasil, v.29, p.177$183,2000$.

Setzer, J. Atlas climático e ecológico do Estado de São Paulo. São Paulo: Centrais Elétricas deSão Paulo-CESP,1966.61p.

SPONGOSKI, S.; ReIS, P.R.; ZaCARIaS, M.S. Acarofauna da cafeicultura de cerrado em Patrocínio, Minas Gerais. Ciência e Agrotécnica, v.29, p.9-17, 2005.

Thomaziello, R.A.; Fazuoli, L.C.; Pezzopane, J.R.M.; Fahl, J.I.; Carelli, M.L.C. Café arábica: cultura e técnicas de produção. Campinas: Instituto Agronômico de Campinas, 2000. 82p. (Boletim técnico 187).

WALter, D.E. Living on leaves: mites, tomenta, and leaf domatia. Annual Review of Entomology, v.41, p.101-114, 1996.

Walter, D.E. \&; O’DowD, D.J. Leaves with domatia have more mites. Ecology, v.73, p.1.514-1.518, 1992.

Recebido em 19/4/06

Aceito em 1/8/06 